\title{
Longitudinal Tracking with Phase and Amplitude Modulated RF*
}

\author{
D.D. Caussyn ${ }^{a}$, M. Ball ${ }^{a}$, B. Brabson ${ }^{a}$, J. Budnick ${ }^{a}$, A. W. Chao ${ }^{b}$, J. Collins ${ }^{a}$, \\ V. Derenchuk ${ }^{a}$, S. Dutt ${ }^{b}$, G. East ${ }^{a}$, M. Ellison ${ }^{a}$, T. Ellison ${ }^{a}$, D. Friesel ${ }^{a}$, B. Hamilton ${ }^{a}$, \\ H. Huang ${ }^{a}$, W.P. Jones ${ }^{a}$, S.Y. Lee ${ }^{a}$, D. Li ${ }^{a}$, M.G. Minty ${ }^{c}$, S. Nagaitsev ${ }^{a}$, K.Y. Ng ${ }^{e}$, \\ X. Pei ${ }^{a}$, T. Sloan ${ }^{a}$, M. Syphers ${ }^{b}$, Y. Wang ${ }^{a}$, Y.T. Yan ${ }^{b}$, P.L. Zhang
}

\section{Abstract}

Synchrotron motion was induced by phase shifting the rf of the Indiana University Cyclotron Facility (IUCF) cooler-synchrotron. The resulting coherent-bunch motion was tracked in longitudinal phase space for as many as 700000 turns, or for over $\mathbf{3 5 0}$ synchrotron oscillations. Results of recent experimental studies of longitudinal motion in which the of phase and amplitude were harmonically modulated are also presented. Comparisons of experimental data with numerical simulations, assuming independent particle motion, are made. Observed multiparticle effects are also discussed.

\section{Introduction}

The IUCF cooler storage ring and synchrotron accelerator was the first of many similar accelerator storage rings designed specifically to employ electron cooling to produce and use high quality medium energy ion beams for nuclear research [1]. This machine has also been a near ideal laboratory for conducting accelerator physics experiments. For a beam of $45 \mathrm{MeV}$ protons the equilibrium $95 \%$ emittance or phase space area is about $0.3 \pi \mathrm{mm}$ mrad with a relative momentum spread full width at half maximum, FWHM, of about 0.0001 . The motion of a beam bunch with this small an emittance can closely simulate single particle motion. Several experiments studying transverse motion near betatron resonances[2] have demonstrated this advantage. In the last year, we have applied many of the same techniques for studying transverse motion on a turn-by-turn basis to a study of longitudinal motion, some of which has been reported previously [3].

Since its discovery in 1945 by McMillan and Veksler[4] synchrotron motion has come to be relatively well understood. For a system with time-independent system parameters, the difference equations describing the longitudinal motion are

$$
\begin{aligned}
& \delta_{n+1}=\delta_{n}+\frac{e V}{\beta^{2} E}\left(\sin \phi_{n}-\sin \phi_{0}\right)-2 \alpha \delta_{n} \\
& \phi_{n+1}=\phi_{n}+2 \pi h \eta \delta_{n+1},
\end{aligned}
$$

where $\delta$ is the fractional momentum deviation of a particle from that of a synchronous particle, and $\phi$ is the phase of

\footnotetext{
-This work supported by the National Science Foundation under Grant NSF PHY 9221402. * IUCF, Indiant Univenity, Bloomington, IN 47405; - The SSC laboratory, 2550 Beckleymeade Avenue, Dallas, TX 75237-3946; “ SLAC, MS26, Box 4349, Stanford, CA 94309; ¿ Brookhaven National Laborntory, Upton, NY 11973; • Fermileb, P.O. Box 500, Bataria, IL 60510
}

the particle relative to the $\mathrm{rf}$, with the subscripts on these variables indicating the turn number. In addition, $\eta$ is the phase slip factor, $\phi_{0}$ is the phase of a synchronous particle, which for a stored beam is 0 deg, $h$ is the harmonic number, $\alpha$ is the damping coefficient, $V$ is the of voltage, $e$ is the particle's charge, $E$ is the particle's total energy, and $\beta$ is the usual relativistic factor.

Assuming that the system is not dissipative, these difference equations can be approximated by the differential equations of motion which can be found from the Hamiltonian $\mathbf{H}$ given by

$$
H=\frac{1}{2} h \omega_{0} \eta \delta^{2}+\frac{e V \omega_{0}}{2 \pi \beta^{2} E}\left[\left(\cos \phi-\cos \phi_{0}+\left(\phi-\phi_{0}\right) \sin \phi_{0}\right)\right]
$$

where $\omega_{0}$ is the particle revolution frequency. While the difference equations provide a more exact description of the motion, Hamiltonian mechanics can be useful for gaining insight on the motion. Even when the beam is electron cooled, the damping is small enough that the approrimations in using Hamiltonian mechanics still result in a reasonable deseription of the motion.

We have experimentally tracked the beam in ten-turn intervals, and made comparisons of the observed motion with the theory. The response of the beam due to modulating the phase and the voltage of the if, was also easily measured using the same techniques.

\section{Procedure and Results}

The experiment began with a beam bunch stored in the IUCF cooler ring having about $3 \times 10^{8}$ protons with $\mathrm{k}$ netic energy of about $45 \mathrm{MeV}$. The IUCF cooler ring has a circumference of $86.82 \mathrm{~m}, \eta$ was about -0.86 , and we were using an if system with a frequency of $1.03148 \mathrm{MH}$ s with a harmonic number $h$ of one. The beam bunch was about $60 \mathrm{~ns}$ (or $5.4 \mathrm{~m}$ ) FWHM. The beam was injected into the cooler ring in a $10 \mathrm{~s}$ cycle, with injection and electron cooling being completed within the first $5 \mathrm{~s}$.

Since measurements of longitudinal motion were being made, the phase lock feedback loop for the $\mathrm{r}$, which is normally on, was switched off. Phase shifting and phase modulation of the beam relative to the $\mathrm{rf}$ was achieved by phase shifting or modulating the rf control signal. The of cavity had a $Q$ value of about 40 at a frequency of $1 \mathrm{MHs}$, with a resulting half-power bandwidth of $25 \mathrm{kHz}$ so the response to a step phase shift occurs in about 40 turns, which is relatively short compared to the shortest synchrotron period used of about 1920 turns.

The phase of the beam was determined from the relative phase between the signal from a pickup coil in the rf cavity, 


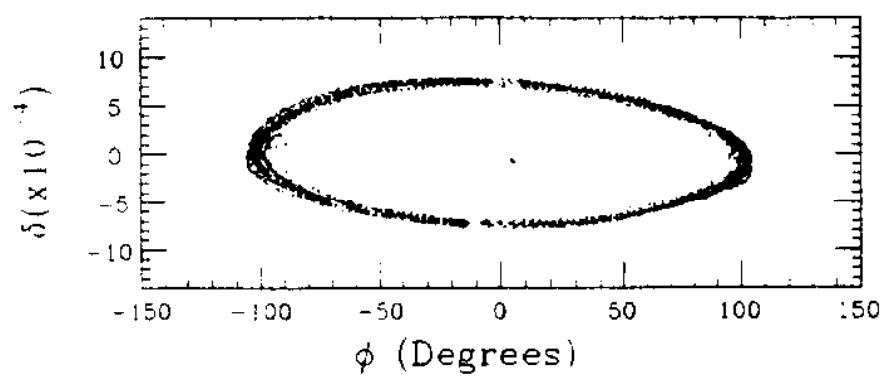

Figure 1: A longitudinal phese space plot after inducing s synchrotron oscillation by making a sudden shift in the of phase. The asymmetry observed here is due to a defect in the phase detector used.

and the sum signal from a beam position monitor (BPM) after it had been passed through a $1.4 \mathrm{MHz}$ low pass filter. The work being reported here was conducted over a period of time in which three different types of phase detectors [5] were used. The first two phase detectors had either an inadequate range of operation, or an uneven response over its full range. Our final phase detector had a range of 720 deg and replaced the first two.

The momentum deviation of the beam was found from the deviation of the beam from the closed orbit $\Delta x_{\mathrm{co}}$ measured by a BPM in a region of high dispersion $D_{2}$. The fractional momentum deviation $\delta$, could then be found from $\delta=\Delta x_{\text {co }} / D_{\text {z }}$ where $D_{\mathrm{z}}$ was measured to be $3.9 \mathrm{~m}$. The position signal was passed through a $3 \mathrm{kHz}$ low pass filter to remove the effects of any coherent betatron oscillations.

Both the $\delta$ and phase signals were digitized using our data aquisition system which has been described elsewhere[2]. As many as 16384 points were digitized in time intervals which were integer multiples of the beam revolution period. A typical measurement of the longitudinal phase space variables after inducing a synchrotron oscillation is shown in Fig. 1 as a Poincaré plot, where every tenth turn is plotted. Similar data was taken for induced synchrotron oscillations having initial phase amplitudes in the range from 10 to 150 deg measured in fiftyturn intervals. The synchrotron tune $\nu_{n}$, which is the ratio of the synchrotion frequency to the revolution frequency, was determined for each case from a fast-Fourier transform (FFT), or from a measurement of the synchrotron period and was found to shift with amplitude as expected from theory[3]. This data was also used to estimate the damping time for the synchrotron oscillation due to the electron cooling. The $1 / e$ damping time was found to be about $400 \mathrm{~ms}$, which corresponds to a value of $\alpha$ in Eq.(1) of about $2.5 \times 10^{-6}$.

The longitudinal response of the beam to forced phase oscillations was also studied. In this case an additional term $\Delta \phi_{\mathrm{rt}}$ must be included in Eq. (2) where $\Delta \phi_{\mathrm{rf}}=$ $a\left(\sin \omega_{m} t_{n+1}-\sin \omega_{m} t_{n}\right)$ with a the phase modulation amplitude, $\omega_{m}$ the angular frequency of the rf phase modulation, and $t_{n}$ and $t_{n+1}$ the beam arrival times at the $\mathrm{rf}$

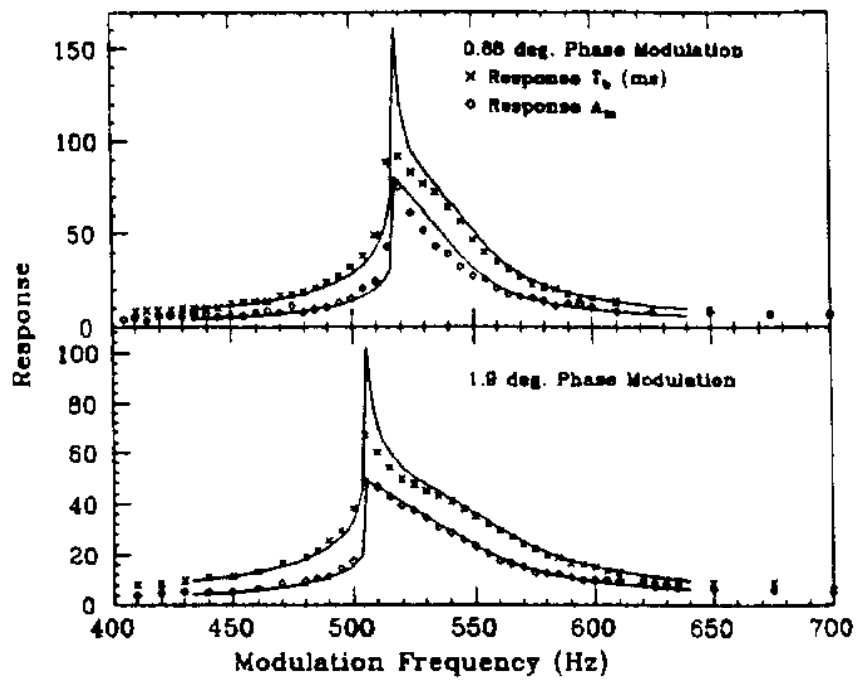

Figure 2: A plot of the measured beam response to phane modulation for two different phase modulation amplitudes. The solid lines are the results from a numerical aimulntion using Eqs. 1 and 2.

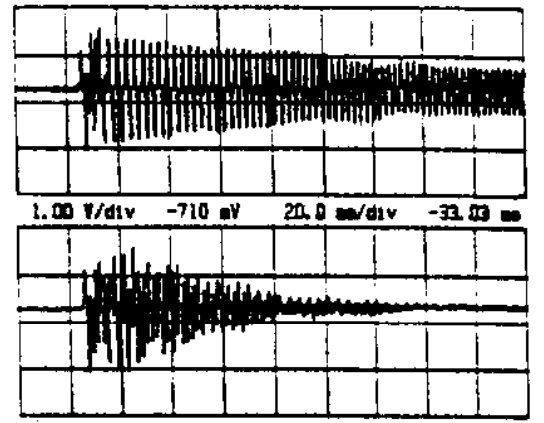

Figure 3: A plot thowing the $\phi$ signal on a digital oscilloscope. The upper trace is with the deetron-cooling "on", and the lower trace is with the cooling "off".

carity, which are approximately multiples of the revolution period for a synchronous particle. Experimentally this was accomplished by modulating the phase of the of with various $a$ and $\omega_{m}$. The transient response was then measured.

The amplitude response $A_{m}$ was found by measuring the peak phase amplitude and normalizing it by dividing by $a$. The frequency response was characterized by measuring the beat period $T_{m}$ of the phase signal. In Fig. 2, the measured responses as a function of phase modulation frequency are plotted for two different phase modulation amplitudes. The synchrotron frequency was about $536 \mathrm{~Hz}$ in this case. The sudden transition from the lower stable solution to the upper stable solution near a frequency called the bifurcation frequency is evident in this figure $[3,6]$. This is a characteristic shared by many other parametric resonant systems[7].

An interesting aspect of these measurements was the relative insignificance of decoherence when the electron cooling was optimized. In Fig. 3 , the phase amplitude as a function of time is shown for two cases where the applied phase shift was about $150 \mathrm{deg}$. In the first case the electron energy was optimized to maximize cooling. In the 


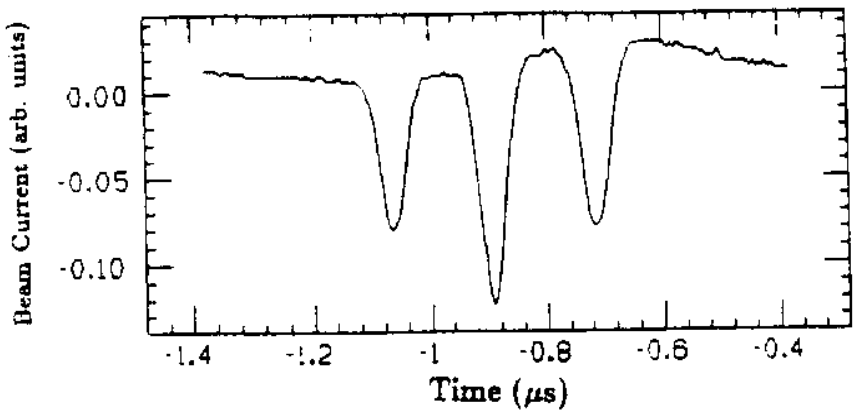

Figure 4: Equilibrium bunch shape for $5 \%$ roltage modaletion where $\nu_{m}$ is $4.75 \times 10^{-4}$.

second case, the electron energy was shifted $20 \mathrm{~ms}$ prior to the start of the measurement so that electron cooling would be negligible (the electron velocity was shifted by about $0.5 \%$ ). Note that the damping of the coherent signal due to decoherence in the absence of electron cooling is relatively rapid.

The response of the beam to rf voltage modulation was also investigated. At this time, we chose to study the equilibrium or steady-state response of the beam after the transient had time to damp out. Experimentally, this was accomplished by modulating the reference voltage in the automatic level control (ALC) circuit for the of voltage by some fractional amount $\epsilon$. The motion in this case is described by the difference equations after multiplying $V$ in Eq.(1) by $1-\epsilon \sin \omega_{m} t$. The revolution frequency in this case was $1.03168 \mathrm{MHz}$ and the small amplitude synchrotron tune $\nu_{10}$ (without voltage modulation) was $2.55 \times 10^{-4}$.

We found that when the beam was allowed to reach equilibrium, it frequently split into multiple bunches in a process that could take several seconds to occur. It was also observed that the degree to which this occurred depended on the beam current. In Fig. 4, the BPM sum signal trace from a fast digital oscilloscope is shown for a modulation tune of $4.75 \times 10^{-4}$ with the if voltage modulated by $5 \%$. By making an appropriate canonical transformation to a rotating reference frame, Hamiltonian methods reveal that the particles accumulated at the locations of stable fixed points (SFP) in this rotating reference frame[8].

Since the various phase detectors we employed were not intended for use with muitiple bunches, we used an oscilloscope to measure the peak phase ampiitude for the response. The measured responses, as maximum phase, are plotted in Fig. 5. The large responses were obtained by starting the voltage modulation at injection where the beam occupies a larger amount of phase space. The gap in the measured response for $\nu_{m}$ from $4.91 \times 10^{-4}$ to $5.02 \times 10^{-4}$ occurred due to the small separation of the SFP's compared to the full width of the particle distribution of about $40 \mathrm{deg}$. For $\nu_{\mathrm{m}}$ above about $5.02 \times 10^{-4}$, the response could again be determined since the SFP at the origin becomes an unstable fixed point (UFP), producing a greater separation between the remaining SFP's. The

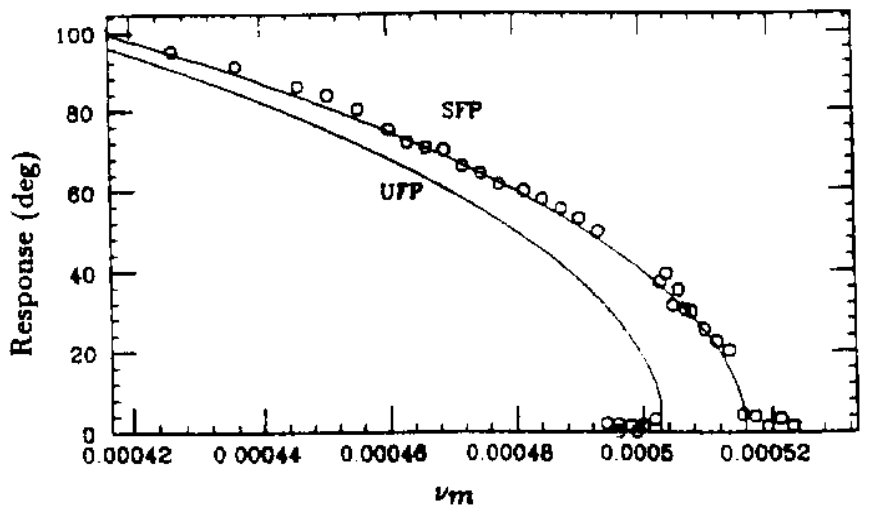

Figure 5: Beam response for $5 \%$ roltege modulation.

solid lines in Fig. 5 correspond to the maximnm phases of the observed SFP's and of a nearby UFP[8].

\section{Conclusions}

We have found that the experimental techniques used for turn-by-turn tracking in studying transverse motion are equally effective for studying single-particle longitudinal motion, at least in the case of transient motion. The steady-state motion was somewhat more difficult to study. In this case, the beam bunch may separate into two or more bunches within an if bucket. Phase space tracking of individual bunches then becomes difficult. Multiparticle aspects of the motion we have observed are interesting in their own right. The rate at which the bunch splitting occurs, the dependence of the amount of beam accumulating at each SFP on the beam current, and the relationship between decoherence and electron cooling may be of practical interest in schemes in which these effects are to be used for slow particle extraction, or in trying to understand bunch diffusion.

\section{References}

[1] R.E. Pollock, Proc. 1989 IEEE P.A.C., Acc. Eng. and Tech., Chicago, IL, p. 17; D.L. Friesel, T.J. Ellison, and P. Schwandt, Nucl. Inst. Meth. B40/41 927 (1989); Timothy JP Ellison,Dennis L. Friesel, and Robert J. Brown, ibid, pp. 633-635; T. Ellison, "Electron Cooling", Indiana University, Bloomington, Indiane, Ph.D. Dissertation, 1990 (unpublished).

[2] S.Y. Lee, et al., Phys. Rev. Lett. 67, 3768 (1991); D.D. Caussyn, et al., Phys. Rev. A 46, 7942 (1992).

[3] M. Ellison, et al., Phys. Rev. Lett., Phys. Rev. Lett. $70591,(1993)$.

[4] E.M. McMillan, Phys. Rev., 68, 143 (1945); V.I. Veksler, Compt. Rend. Acad. Sci. U.S.S.R., 43, 329 (1944); ibid. 44, 365 (1944).

[5] Roland E. Best, Phase Locked Loops, Theory, Design, and Applications (McGraw-Hill, 1984) pp. 7-9.

[6] H. Huang, et al., to be published.

[7] D.D'Humieres, M.R. Beasley, B.A. Huberman, and Libchaber, Phys. Rev. A 26, 3483 (1982).

[8] $\mathrm{D} . \mathrm{Li}$, et al., to be published. 